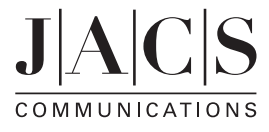

Published on Web 01/17/2006

\title{
Springs, Rings, and Spirals of Rutile-Structured Tin Oxide Nanobelts
}

\author{
Rusen Yang ${ }^{\dagger}$ and Zhong Lin Wang ${ }^{*,+, \ddagger}$ \\ School of Materials Science and Engineering, Georgia Institute of Technology, Atlanta, Georgia 30332-0245, and \\ National Center of Nanoscience and Technology, and College of Engineering, Peking University, Beijing, China
}

Received November 16, 2005; E-mail: zhong.wang@mse.gatech.edu

Synthesis of nanomaterials with well-controlled size, morphology, and chemical composition may open new opportunities in exploring a material's chemical and physical properties. Since the discovery of semiconducting oxide nanobelts ${ }^{1}\left(\mathrm{ZnO}, \mathrm{SnO}_{2}, \mathrm{CdO}\right.$, $\mathrm{Ga}_{2} \mathrm{O}_{3}$, and $\mathrm{PbO}_{2}$ ) in 2001, nanobelts have been found and widely investigated in numerous materials including but not limited to ${ }^{2}$ ( $\mathrm{Zn}, \mathrm{Si}$, and C) II-IV semiconductors ${ }^{3}(\mathrm{ZnS}, \mathrm{CdSe}$, and $\mathrm{ZnSe})$ and some other compounds ${ }^{1,4}\left(\mathrm{In}_{2} \mathrm{O}_{3}, \mathrm{Ge}_{3} \mathrm{~N}_{4}, \mathrm{Bi}_{2} \mathrm{~S}_{3}, \mathrm{SiC}, \mathrm{GaP}\right.$, and $\mathrm{Pb}_{3} \mathrm{O}_{2} \mathrm{Cl}_{2}$ ). Among these materials, wurtzite-structured $\mathrm{ZnO}$ is the most outstanding member. The uniqueness of the wurtzite structure is its noncentral symmetry and the surface polar charges due to the cation- and anion-terminated surfaces, such as $\mathrm{Zn}^{2+}$-terminated $(0001)$ and $\mathrm{O}^{2-}$-terminated $(000 \overline{1})$. To minimize the electrostatic interaction energy among the polar charges, the nanobelt dominated by the $\pm(0001)$ polar surfaces tends to fold over, resulting in the formation of single-crystalline nanorings, nanosprings, and nanospirals. ${ }^{5}$ The reduction in the area of polar surfaces causes a structural transformation of a single-crystal $\mathrm{ZnO}$ nanobelt into a superlattice-structured, partial-polar surface dominated nanobelt, which eventually forms a rigid nanohelix. ${ }^{6}$ Besides the nanorings observed for wurtzite AlN due to polar surfaces, ${ }^{7}$ no report has been found on the formation of single-crystalline rings/springs/ spirals for a material that has a structure different from wurtzite.

In this paper, we report for the first time the discovery of singlecrystalline $\mathrm{SnO}_{2}$ springs, rings, and spirals, which have the rutile structure. The grown nanostructures are made of tetragonal $\mathrm{SnO}_{2}$ with the (011) polar surfaces facing toward the center. The formation process has been analyzed based on the polar charge interaction model. The discovery of $\mathrm{SnO}_{2}$ rings and springs presents another family of polar surface dominated growth phenomena in a nonwurtzite-structured material, indicating the possibility of forming similar structures for other materials, such as $\mathrm{TiO}_{2}$. Considering the important application of $\mathrm{SnO}_{2}$ in sensors, these structures are not only ideal systems for fundamental understanding of the polarization effect on the morphology at the nanoscale level, but they also have potential applications ${ }^{8}$ as nanoscale sensors, resonators, and transducers.

$\mathrm{SnO}_{2}$ nanostructures were synthesized through a solid-vapor process in a horizontal tube furnace. The experimental apparatus has been described elsewhere. ${ }^{9}$ After the tube had been evacuated to a pressure of $1 \times 10^{-3}$ Torr, commercial SnO powders (Alfa Aesar) in the middle of the tube were heated to $800{ }^{\circ} \mathrm{C}$, held for $90 \mathrm{~min}$, heated again up to $1100{ }^{\circ} \mathrm{C}$ and held for another $50 \mathrm{~min}$ to complete the synthesis of the $\mathrm{SnO}_{2}$ nanostructures. The Ar carrier gas was flowed through the system at a rate of $50 \mathrm{sccm}$ (standard cubic centimeters per minute) under a constant pressure of $300 \mathrm{mbar}$ during the entire synthesis process. The grown nanostructures were collected using a polycrystalline $\mathrm{Al}_{2} \mathrm{O}_{3}$ substrate placed downstream in the tube furnace at a temperature range of $600-700{ }^{\circ} \mathrm{C}$.

$\dagger$ Georgia Institute of Technology.

* National Center of Nanoscience and Technology and Peking University

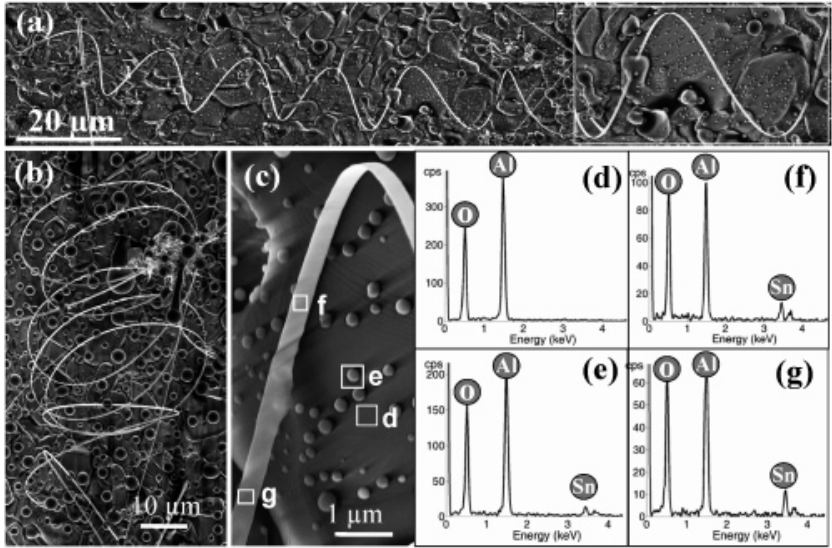

Figure 1. Scanning electron microscopy (SEM) and energy-dispersive $\mathrm{X}$-ray spectroscopy (EDS) of $\mathrm{SnO}_{2}$ nanostructures. (a) SEM image of a $\mathrm{ZnO}$ nanospring. (Inset) An enlarged image of the nanospring. (b) SEM image of $\mathrm{SnO}_{2}$ nanospirals. (c) A higher magnification SEM image from the nanospring indicated in (a). (d-g) EDS spectra acquired from the areas labeled with $\mathrm{d}, \mathrm{e}, \mathrm{f}$, and $\mathrm{g}$ in panel $\mathrm{c}$, respectively.

We first examined the as-synthesized product by scanning electron microscope (SEM) in conjunction with energy-dispersive X-ray spectroscopy (EDS). We found that $\mathrm{SnO}_{2}$ nanobelts tend to bend while they grow longitudinally. As a result, numerous $\mathrm{SnO}_{2}$ spirals formed on the substrate. In addition, springs and rings were also found in the grown product. Figure 1a shows a $\mathrm{SnO}_{2}$ spring lying on an $\mathrm{Al}_{2} \mathrm{O}_{3}$ substrate. The helical structure is made of a uniformly curved $\mathrm{SnO}_{2}$ nanobelt with a pitch distance of $\sim 20 \mu \mathrm{m}$. The most common $\mathrm{SnO}_{2}$ spirals are shown in Figure 1b, in which the curvature of the $\mathrm{SnO}_{2}$ nanobelt changed during its growth. Most spirals/rings have diameters of $10-50 \mu \mathrm{m}$. A magnified part of the spring from Figure 1a is given in Figure 1c, showing clearly a belt-like geometry with a width of $\sim 300 \mathrm{~nm}$ and a thickness of several tens of nanometers. In addition, numerous nanoparticles are also formed on the $\mathrm{Al}_{2} \mathrm{O}_{3}$ substrate. To investigate the chemical composition of the product, we took EDS spectra from a series of locations along the nanobelt, on the nanoparticles, and on the blank substrate, as labeled from $\mathrm{d}-\mathrm{g}$ in Figure $1 \mathrm{c}$, and the results are displayed in Figure 1d-g, respectively. The EDS spectrum in Figure $1 \mathrm{~d}$ shows only $\mathrm{Al}$ and $\mathrm{O}$ peaks with an atomic ratio close to 2:3, which is consistent with the composition of the underneath $\mathrm{Al}_{2} \mathrm{O}_{3}$ substrate. The absence of a $\mathrm{Sn}$ peak or any other peaks excludes a possible layer of $\mathrm{SnO}_{2}$ or $\mathrm{Sn}$ that might be formed on the substrate surface. After deducting the $\mathrm{Al}_{2} \mathrm{O}_{3}$ component, the EDS spectra in panels $\mathrm{f}$ and $\mathrm{g}$ indicate that the nanobelt is composed of elements Sn and O.

The crystal structure of the $\mathrm{Sn}-\mathrm{O}$ nanobelt was determined with transmission electron microscopy (TEM) and selected area electron diffraction (SAED). The TEM image in Figure 2a presents a full ring formed by a closed nanobelt. The single-crystalline nature is confirmed by the sharp SAED patterns along the constituent 


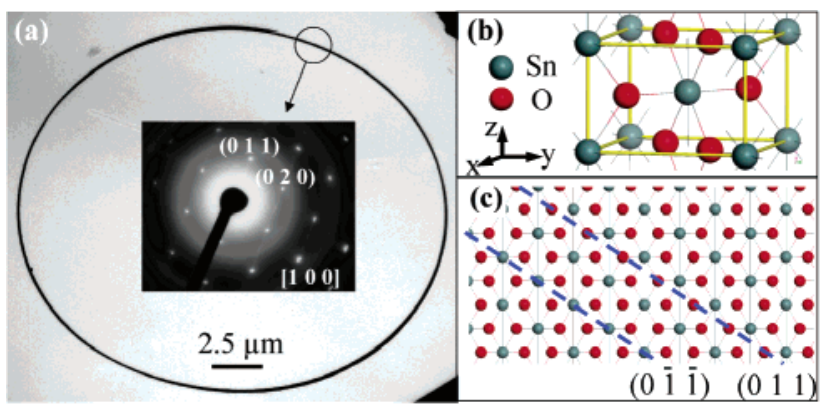

Figure 2. (a) Low magnification TEM image of a $\mathrm{SnO}_{2}$ ring and the corresponding SAED pattern (inset) from the circled region. (b) Tetragonal structure model of $\mathrm{SnO}_{2}$. (c) The structure model of $\mathrm{SnO}_{2}$ projected along [100], displaying the $\pm(011)$ polar surfaces.

nanobelt. The SAED pattern in the inset of Figure $2 b$ was taken along the direction normal to the plane of the ring. Analysis of the SAED pattern reveals that the nanobelt has a tetragonal crystal structure with lattice parameters $a=b=4.75 \AA$ and $c=3.20 \AA$, which are consistent with the lattice parameters of the rutile $\mathrm{SnO}_{2}$ ( $P 4_{2} / \mathrm{mnm}, a=b=4.7382 \AA$ and $c=3.1871 \AA$, JPCDS No. 411445). It is noteworthy that the $\mathrm{SnO}_{2}$ belt grows along $\sim[0 \overline{1} 1]$ with the side surfaces facing the center of the ring being $\pm(011)$ and the top and bottom surfaces parallel to the ring plane being \pm (100). SAED and TEM examination of several other $\mathrm{SnO}_{2}$ nanostructures consistently shows that the nanobelt is dominated by the $\pm(011)$ surfaces.

The growth of the ring, spring, and spirals can be understood on the basis of polar surfaces of the rutile-structured $\mathrm{SnO}_{2}$, the atomic model of which is shown in Figure $2 b$. By projecting the structure model along [100], as shown in Figure 2c, the $\pm(011)$ plane can be terminated either solely with $\mathrm{Sn}^{4+}$ or solely with $\mathrm{O}^{2-}$, respectively, resulting in a pair of positively and negatively charged polar surfaces on the inner and outer surfaces (or vice versa) of the nanobelt. During the growth at $\sim 500-600{ }^{\circ} \mathrm{C}$ in an argon atmosphere, the probability for foreign molecules being adsorbed on the surfaces was negligible within the time of the growth; thus, the polar charges are likely to be preserved. If the charges on the $\pm(011)$ side surfaces are not compensated during the growth, the net dipole moment across the side surfaces and the surface energy tend to diverge with an increase of the nanobelt length. On the other hand, the electrostatic energy due to the dipole moment can be minimized or neutralized when the nanobelt rolls up to form a ring or spiral structure. On the other hand, bending of the nanobelt introduces elastic deformation energy, which increases with the decrease of the ring/spiral radius. The minimization of the total energy contributed by electrostatic polarization energy and elastic deformation energy determines the final morphology of the $\mathrm{SnO}_{2}$ nanobelt, resulting in the formation of the ring (Figure $2 \mathrm{a}$ ) or multiply looped spiral (Figure 1b). Alternatively, if the bending of the nanobelt proceeds during the growth along the axial direction of the curled nanobelt, a helical structure will form (Figure 1a). The spring is formed due to the stretching of the spiral (or multilooped ring) and/or possibly the electrostatic repulsion between the two adjacent rings.

The above proposed mechanism is consistent with the model established for $\mathrm{ZnO} .{ }^{5} \mathrm{In}$ comparison, the $\mathrm{ZnO}$ nanorings/nanosprings reported previously have radii of $\sim 500-800 \mathrm{~nm}$, a nanobelt width of $10-60 \mathrm{~nm}$, and a thickness of 5-20 nm. The radii of the $\mathrm{SnO}_{2}$ rings and springs reported in this paper are 5-50 $\mu \mathrm{m}$, and the $\mathrm{SnO}_{2}$ nanobelts have widths of a few hundred nanometers and thickness of a few tens of nanometers, which are also larger than those of the $\mathrm{ZnO}$ nanobelts. This may be closely related to the charge density on the polar surfaces. The elastic energy increases rapidly with the curvature, width, and thickness of the nanobelt. Accordingly, it is rational to expect much larger helical nanostructures of $\mathrm{SnO}_{2}$ than that of $\mathrm{ZnO}$. In addition, the surface energies of Sn- and Oterminated surfaces can also contribute to the formation of rings and springs. A deep insight on the effect of polarization, elasticity, and surface energy on the nanostructures requires further investigation with first principle computation and more experiments.

In summary, single-crystalline springs, rings, and spirals have been discovered for the first time in a rutile-structured $\mathrm{SnO}_{2}$. The formation of these nanostructures is mainly due to polar surfaces of tetragonal $\mathrm{SnO}_{2}$. The synthesized $\mathrm{SnO}_{2}$ nanostructures are very important for studying the effect of polar surfaces on the morphology of the nanostructures. The research demonstrates that hierarchical nanostructures, which have been observed previously only for polar surface dominated wurtzite $\mathrm{ZnO}$, are also possible in other materials with different crystal structures. Those hierarchical nanostructures may useful for nanoconductors, transparent electrodes, and gas sensors.

Acknowledgment. The research was supported by NSF, the NASA Vehicle Systems Program and Department of Defense Research and Engineering (DDR\&E), the Defense Advanced Research Projects Agency (N66001-04-1-8903), Chinese Academy of Sciences, and the "973" project.

Supporting Information Available: More SEM and TEM characterizations of the $\mathrm{SnO}_{2}$ nanobelt. This material is available free of charge via the Internet at http://pubs.acs.org.

\section{References}

(1) Pan, Z. W.; Dai, Z. R.; Wang, Z. L. Science 2001, 291, 1947-1950.

(2) (a) Wang, Y.; Zhang, L.; Meng, G.; Liang, C.; Wang, G.; Sun, S. Chem Commun. 2001, 24, 2632-2633. (b) Shi, W.; Peng, H.; Wang, N.; Li, C. P.: Xu, L.; Lee, C. S.; Kalish, R.; Lee, S. T. J. Am. Chem. Soc. 2001 123, 11095-11096. (c) Kang, Z.; Wang, E.; Mao, B.; Su, Z.; Gao, L.; Lian, S.; Xu, L. J. Am. Chem. Soc. 2005, 127, 6534-6535.

(3) (a) Ma, C.; Moore, D.; Li, J.; Wang, Z. L. Adv. Mater. 2003, 15, 228231. (b) Jiang, Y.; Meng, X. M.; Liu, J.; Xie, Z. Y.; Lee, C. S.; Lee, S. T. Adv. Mater. 2003, 15, 323-327. (c) Ma, C.; Ding, Y.; Moore, D. Wang, X.; Wang, Z. L. J. Am. Chem. Soc. 2004, 126, 708-709. (d) Jiang, Y.; Meng, X. M.; Yiu, W. C.; Liu, J.; Ding, J. X.; Lee, C. S.; Lee, S. T. J. Phys. Chem. B 2004, 108, 2784-2787. (e) Johnson, J. C.; Knutsen, K. P.; Yan, H.; Law, M.; Zhang, Y.; Yang, P.; Saykally, R. J. Nano Lett. 2004, 4, 197-204.

(4) (a) Gao, Y. H.; Bando, Y.; Sato, T. Appl. Phys. Lett. 2001, 79, 45654567. (b) Liu, Z.; Peng, S.; Xie, Q.; Hu, Z.; Yang, Y.; Zhang, S.; Qian, Y. Adv. Mater. 2003, 15, 936-940. (c) Xi, G.; Peng, Y.; Wan, S.; Li, T.; Yu, W.; Qian, Y. J. Phys. Chem. B 2004, 108, 20102-20104. (d) Tsai, J. S.; Chen, F. R.; Kai, J. J.; Chen, C. C.; Huang, R. T.; Wang, M. S.; Huang, G. C.; Guo, G. G.; Yu, M. U. J. Appl. Phys. 2004, 95, $2015-$ 2019. (e) Sigman, M. B.; Korgel, B. A. J. Am. Chem. Soc. 2005, 127, $10089-10095$

(5) (a) Kong, X. Y.; Wang, Z. L. Nano Lett. 2003, 3, 1625-1631. (b) Kong, X. Y.; Wang, Z. L. Appl. Phys. Lett. 2004, 84, 975-977. (c) Kong, X. Y.; Ding, Y.; Yang, R.; Wang, Z. L. Science 2004, 303, 1348-1351. (d) Yang, R.; Ding, Y.; Wang, Z. L. Nano Lett. 2004, 4, 1309-1312.

(6) Gao, P. X.; Ding, Y.; Mai, W.; Hughes, W. L.; Lao, C. S.; Wang, Z. L. Science 2005, 309, 1700-1704.

(7) Duan, J. H.; Yang, S. G.; Liu, H. W.; Gong, J. F.; Huang, H. B.; Zhao, X. N.; Tang, J. L.; Zhang, R.; Du, Y. W. J. Cryst. Growth 2005, 283 , $291-296$.

(8) Maiti, A.; Rodriguez, J. A.; Law, M.; Kung, P.; McKinney, J. R.; Yang, P. Nano Lett. 2003, 3, 1025-1028.

(9) Dai, Z. R.; Pan, Z. W.; Wang, Z. L. J. Am. Chem. Soc. 2002, 124 $8673-8680$.

JA0578000 